\title{
Nuevas perspectivas en el tratamiento de la Esclerosis múltiple
}

\author{
Arcos Sánchez C. ${ }^{1}$, Salinas Vela FT. ${ }^{2}$, Olmedilla González MN. ${ }^{3}$
}

Sanid. mil. 2011; 67 (2): 108-114; ISSN: 1887-8571

\section{RESUMEN}

El conocimiento de la etiopatogenia y el pronóstico de la Esclerosis Múltiple (EM) ha permitido desarrollar tratamientos nuevos y más eficaces. Las terapias actuales pueden dividirse en tres grandes grupos: los tratamientos del brote agudo, los modificadores del curso de la enfermedad y el tratamiento sintomático. Actualmente hay seis fármacos aprobados para el tratamiento de la EM en España, dos nuevos fármacos orales (Cladribina y Fingolimod) y otros destinados a mejorar la calidad de vida de los pacientes (Fampridina y Sativex) están pendientes de aprobación y varios en distintas fases de estudios clínicos que ofrecen perspectivas muy esperanzadoras.

PALABRAS CLAVE: Esclerosis múltiple, tratamiento, terapia farmacológica.

\section{New perspectives in the treatment of Multiple Sclerosis}

\section{SUMMARY}

A best knowledge of etiopathogeny and prognosis of Multiple Sclerosis is allowing the development of new and more effective therapies. Current therapies are divided into three groups: treatments for the acute relapses, disease-modifying therapy and symptomatic treatment. There are six approved drugs available in Spain, also there are two oral new drugs (Cladribina and Fingolimod) and others designed for improving the quality of life of these patients (Frampidina and Sativex) waiting to be approved and some others in different stages of clinical trials that will offer encouraging perspectives in a near future.

KEY WORDS: Multiple sclerosis, Treatment, Drug therapy.

\section{INTRODUCCIÓN}

La Esclerosis Múltiple ${ }^{1}$ es un trastorno crónico, autoinmunitario y neurodegenerativo del sistema nervioso central (SNC) caracterizado por inflamación, desmielinización, pérdida de oligodendrocitos y neuronas, que da lugar a una acumulación de discapacidad neurológica. Continúa siendo una de las principales causas de incapacidad permanente en las personas jóvenes.

La EM es una enfermedad heterogénea en su presentación y evolución. Normalmente la enfermedad se presenta en forma de episodios de déficit neurológico agudo, dando lugar a una forma remitente-recurrente (EMRR) que se caracteriza por brotes (recidivas) seguidos de recuperación completa o incompleta. Con el tiempo, en la mayoría de los pacientes se produce la evolución a un curso secundariamente progresivo (EMSP), que se caracteriza por una progresión continua.

Aproximadamente un 10-15\% de los pacientes con EM presenta un curso primario progresivo (EMPP), definido por un deterioro de las funciones neurológicas desde el inicio de los síntomas sin exacerbaciones o remisiones superpuestas. En un 1-3\% de los casos los pacientes evolucionan de manera rápida en pocos meses tras el inicio de la enfermedad (EM maligna o fulminante).

${ }^{1}$ Cap. Médico. Servicio de Neurología.

2 Cap. Médico. Servicio de Radiodiagnóstico.

${ }^{3}$ Cte. Médico. Servicio de Neurología.

Hospital Central de la Defensa Gómez Ulla. Madrid. España.

Dirección para correspondencia: Dra. Carolina Arcos Sánchez. Hospital Central de la Defensa. Servicio de Neurología. Glorieta del Ejército s/n. 28047 Madrid. E-mail: carcsan@ fn.mde.es

Recibido: 3 de mayo de 2010

Aceptado: 2 de febrero de 2011

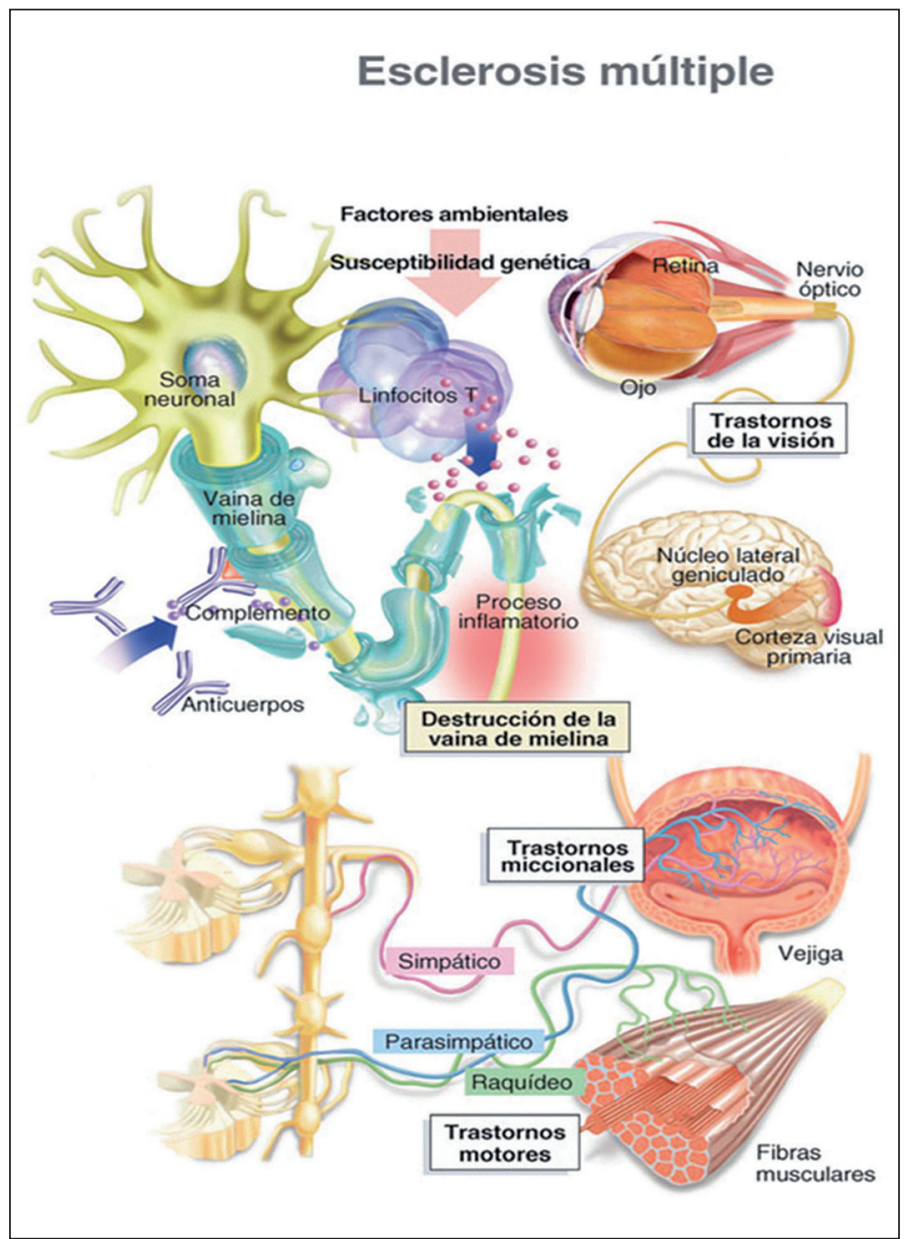

Figura 1. Patogenia de la EM. 
Se denomina Síndrome Clínico Aislado (CIS, por sus siglas en inglés) al primer brote sugestivo de EM, actualmente se inicia el tratamiento tras este primer episodio, ya que el riesgo de progresión a EM Cínicamente Definida (EMCD) es del 82\% en 20 años.

La EM es una enfermedad autoinmunitaria desmielinizante crónica del sistema nervioso central (SNC) mediada por linfocitos T helper $1 \mathrm{CD} 4+$ dirigidos contra antígenos del complejo oligodendrocito-mielina, si bien la participación de otros subtipos celulares, como linfocitos B, monocitos-macrófagos y microglía activada, conduce a la amplificación de la respuesta inflamatoria.

La lesión activa se caracteriza por la infiltración multifocal perivenular de la sustancia blanca del SNC por linfocitos y monocitos-macrófagos, la presencia de microglia activada y la destrucción de la mielina y de los oligodendrocitos formadores de mielina, con presencia de axones parcialmente mielinizados. A este concepto se ha añadido, en base a los estudios neuropatológicos, la presencia, desde las fases iniciales de la enfermedad, de fragmentación de axones y depósito de proteína precursora de amiloide y los estudios de neuroimagen muestran concentraciones reducidas de $\mathrm{N}$-acetilaspartato (marcador de integridad axonal) y atrofia cerebral, esto ha llevado a considerar a la EM como una enfermedad inflamatoria pero también neurodegenerativa.

Este mayor conocimiento de la enfermedad plantea nuevas perspectivas en el diagnóstico y en el tratamiento.

\section{TRATAMIENTO DE LA EM}

La heterogeneidad en la evolución de la enfermedad es reflejo de los diferentes mecanismos inmunopatológicos subyacentes, los cuales van a condicionar la elección de la estrategia terapéutica apropiada.

Cuando hablamos de tratamiento de la $\mathrm{EM}^{2}$ éste incluye: el tratamiento del brote agudo, los fármacos modificadores del curso de la enfermedad ${ }^{3}$ y los fármacos destinados a la mejoría de los síntomas asociados a la enfermedad. El reto sigue siendo encontrar fármacos curativos, con efecto profiláctico o bien fármacos capaces de reparar las alteraciones neurológicas producidas.

No todas las formas de EM cuentan con un tratamiento de eficacia probada, sino únicamente la forma recurrente-remitente y la secundariamente progresiva con brotes. Lo que se persigue con el tra-

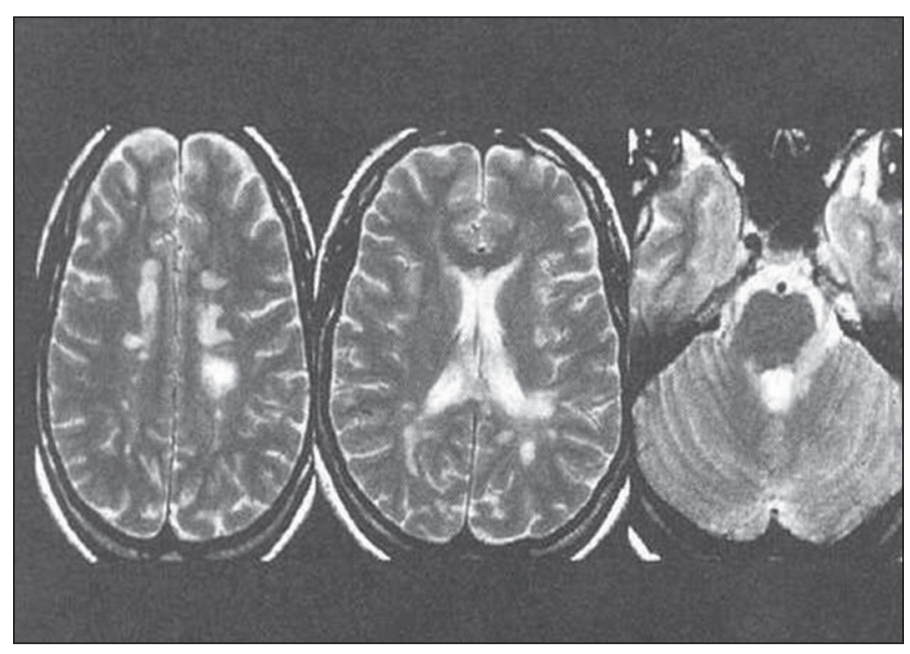

Figura 2. Imagen típica de EM en RMN encefálica. tamiento es reducir el número y la severidad de los brotes y limitar la acumulación de secuelas, sin ocasionar con ello efectos adversos.

En la actualidad se dispone de seis fármacos modificadores del curso de la enfermedad con indicación para EM son tres interferones beta, acetato de glatiramero, natalizumab y mitoxantrona, también está aprobado el uso de azatioprina y de inmunoglobulinas humanas. Estos fármacos pueden dividirse en dos líneas de tratamiento, en la primera línea se encontrarían los interferones y el acetato de glatiramero y en la segunda la mitoxantrona y el natalizumab, probablemente más eficaces pero con mayor índice de efectos adversos potencialmente graves que los primeros que cuentan un razonable perfil de seguridad y tolerancia.

La eficacia de un tratamiento se mide en términos de reducción de la tasa de brotes, de progresión de la enfermedad y en la actividad en Resonancia Magnética Nuclear $(\mathrm{RMN})^{4}$. De estos tres parámetros, la presencia de cambios inflamatorios en RMN es el indicador más sensible de monitorización de la respuesta al tratamiento. Se ha establecido la no eficacia de un tratamiento si a los 6-12 meses existe actividad en RMN. Sin embargo, en los denominados buenos respondedores se evidencia una disminución del volumen lesional en $\mathrm{T} 1$ y T2.

Asimismo en el momento actual se abre una nueva perspectiva en el tratamiento, se están realizando múltiples ensayos clínicos y ya hay fármacos, tanto modificadores del curso de la enfermedad como tratamientos sintomáticos, pendientes de aprobación y cuya comercialización está prevista para el próximo año, que cuentan con una ventaja fundamental: su administración por vía oral. A los que progresivamente se añadirán aquellos que se encuentran en distintas fases de ensayos clínicos.

\section{TRATAMIENTO DEL BROTE AGUDO}

El tratamiento del brote agudo ${ }^{5}$ se basa fundamentalmente en el empleo de megadosis de corticosteroides por vía endovenosa, habitualmente se administra $1 \mathrm{~g}$ al día de 6-metilprednisolona durante 3 o 5 días, que puede estar seguido de una pauta oral descendente. Los glucocorticoides disminuyen la transcripción de citocinas proinflamatorias, regulan la expresión de moléculas de adhesión a la barrera hematoencefálica, reducen el edema tisular y el reclutamiento de células inflamatorias. Lo que se consigue con este tratamiento es acortar la duración de un brote y acelerar la recuperación en pacientes con un primer brote sugestivo de EM o CIS, en EMRR y en la EMSP con brotes.

También se ha utilizado la plasmaféresis, como segunda línea de tratamiento. En un estudio aleatorizado el $42 \%$ de los pacientes que recibieron tratamiento activo presentaron una mejoría funcional importante, frente al 5,9\% de los que recibieron placebo. Solo se usa en casos excepcionales y serían necesarios nuevos estudios controlados y a más largo plazo para comprobar la eficacia real de este tratamiento.

\section{FÁRMACOS MODIFICADORES DEL CURSO DE LA ENFERMEDAD}

Estas terapias son aquellas que han demostrado eficacia para modificar la actividad de la enfermedad, a través de la disminución 
del número y la gravedad de los brotes, y previniendo o retrasando la progresión de la discapacidad neurológica.

Los fármacos incluidos en este grupo, actualmente aprobados son los Interferones, el Acetato de Glatiramero, el Natalizumab, la Mitoxantrona, la Azatioprina y otros inmunosupresores. Estos han demostrado una tasa de disminución de brotes que oscila entre el 18 y el 68\% y una menor progresión de la enfermedad de entre el 12 y el $75 \%$. También encontramos diferencias en cuanto a un enlentecimiento de la evolución radiológica demostrada por resonancia magnética (RM), dato muy importante en cuanto el pronóstico de la enfermedad.

\section{INTERFERONES}

Los interferones ${ }^{6}$ son un grupo de glucoproteínas producidas por numerosas células del organismo a raíz de infecciones virales o de la interacción con superantígenos o mitógenos. Tienen un amplio espectro de efectos antivirales, antiproliferativos e inmunomoduladores, los mecanismos de acción no están bien establecidos; disminuyen la síntesis de citocinas y la expresión de sus receptores, reducen el acceso de células inflamatorias al SNC mediante la inhibición de metaloproteinasas de matriz, efectos sobre la proliferación de células $\mathrm{T}$ y la liberación de IFN-gamma, la migración de células T, la expresión de moléculas del complejo mayor de histocompatibilidad (CMH-II) por parte del IFN-gamma, la expresión de óxido nítrico sintetasa inducible y la expresión del factor neural de crecimiento, entre otros.

Suponen un tratamiento inmunomodulador de primera línea, en EMRR, EMSP y CIS. La eficacia de los IFN-beta ha quedado establecida en varios ensayos clínicos multicéntricos, aleatorizados y controlados con placebo, con una evidencia de clase I.

Los IFN-beta han demostrado ser capaces de reducir la actividad de la enfermedad, tanto clínica, en cuanto a reducción de la tasa de brotes (33\%), como subclínica, en cuanto a carga lesional y actividad en RMN.

Aún es controvertido si una mayor dosis y/o una mayor frecuencia de administración suponen un mayor beneficio terapéutico, los estudios realizados aportan resultados dispares.

El tratamiento con IFN-beta se asocia a la producción de anticuerpos neutralizantes (NAb, por sus siglas en inglés) anti-IFN. La producción de anticuerpos es menor para el IFN-beta 1a intramuscular. Si bien el nivel de evidencia es de clase II, parece que su presencia persistente en suero determina una reducción de la eficacia de los IFN-beta.

Los efectos secundarios son similares, producen fundamentalmente un cuadro pseudogripal caracterizado por fatiga, fiebre y mialgias, el día de la inyección y que va disminuyendo progresivamente durante el tratamiento. Otros efectos secundarios son un aumento leve de las enzimas hepáticas y la linfocitopenia moderada, que rara vez obligan a suspender el tratamiento.

\section{ACETATO DE GLATIRAMERO}

$\mathrm{El}$ acetato de glatiramero $(\mathrm{AG})^{7}$ es el otro inmunomodulador de primera línea aprobado para el tratamiento de la EM. Ha demostrado reducir la actividad de la enfermedad tanto clínica como radiológica, así como retrasar la acumulación de discapacidad.
Tabla 1. Parámetros de RMN que indican presencia de actividad inflamatoria.

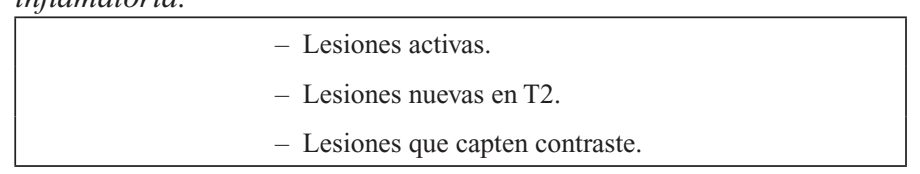

Análogo sintético de la proteína básica de la mielina, se desarrolló para estudiar la biología de la encefalomielitis autoinmune experimental.

No atraviesa la barrera hematoencefálica, se une a moléculas de CMH-I situadas en la superficie de las células presentadoras de antígeno (CPA) que reconocen antígenos de la proteína básica de la mielina (PBM), previniendo la expansión linfocitaria.

Estimula la producción de citocinas antiinflamatorias. También se estudia el posible efecto neuroprotector a nivel de SNC.

Según la literatura no existen diferencias significativas si se compara con el tratamiento con IFN. Se estima que la disminución de tasa de brotes, con ambos tipos de inmunomoduladores, ronda el $29 \%$.

\section{NATALIZUMAB}

El natalizumab ${ }^{8,9}$ es el primer anticuerpo monoclonal aprobado para la EMRR. Se trata de un anticuerpo monoclonal IgG4k humanizado, evita la unión linfocito-endotelio e impide la migración celular a través de la barrera hematoencefálica. Su efecto es inmunosupresor y antiinflamatorio.

Eficacia demostrada en el control de la actividad inflamatoria, el $37 \%$ de los pacientes se encuentran libres de actividad de la enfermedad. La tasa de reducción de brotes es del $68 \%$ y enlentece el empeoramiento de la discapacidad (42\%).

Indicado para formas EMRR que no responden adecuadamente o no toleran otros tratamientos, o en formas agresivas desde el inicio. Se usa en monoterapia. Se administra a dosis de $300 \mathrm{mg}$ cada 28 días por vía endovenosa.

Hasta un $6 \%$ de los pacientes pueden desarrollar anticuerpos persistentes contra el natalizumab, lo que se ha asociado a una disminución de la eficacia y a un incremento de los efectos secundarios.

Los efectos secundarios más frecuentes son cefalea, fatiga y congestión nasal. Tras un breve periodo de comercialización en Estados Unidos, el natalizumab se retiró del mercado al conocerse la aparición de tres casos de leucoencefalopatía multifocal progresiva (LMP), se volvió a aprobar en junio de 2006. A fecha 31 de diciem-

Tabla 2. Fármacos aprobados en España para el tratamiento de la EM.

\begin{tabular}{|lll|}
\hline \multicolumn{1}{|c}{ Fármaco } & Especialidad & \multicolumn{1}{c|}{ Dosis recomendada } \\
\hline Acetato de glatiramero & Copaxone $^{\circledR}$ & $20 \mathrm{mg}$ s.c. 1 vez al día. \\
Interferón beta-1 a & Rebif $^{\circledR}$ & $22 / 44 \mu \mathrm{g}$ s.c. 3 veces por semana. \\
& Avonex $^{\circledR}$ & $20 \mu \mathrm{g}$ i.m. 1 vez por semana. \\
Interferón beta-1b & Betaferon $^{\circledR}$ & $8 \mathrm{MU}$ s.c. en días alternos. \\
& Extavia $^{\circledR}$ & $0,25 \mathrm{mg}$ s.c. 1 vez al día \\
Mitoxantrona & Novantrone $^{\circledR}$ & $12 \mathrm{mg} / \mathrm{m}^{2} 1$ vez cada 3 meses. \\
Natalizumab & Tysabri $^{\circledR}$ & $300 \mathrm{mg}$ i.v. cada 28 días. \\
Azatioprina & Imurel $^{\circledR}$ & $0,3 \mathrm{mg} / \mathrm{kg} /$ día v.o. \\
\hline
\end{tabular}


Tabla 3. Fármacos pendientes de aprobación en España para el tratamiento de la EM.

\begin{tabular}{|llll|}
\hline Uso previsto & Fármaco & Especialidad & Dosis recomendada \\
\hline Tratamiento sintomático & Nabiximol & Sativex & \\
& Fampridina & No comerc. & Pulverización sublingual 4-24 al día. \\
Modificadores & Cladribina & Leustatin & 1 comp. $10 \mathrm{mg} / 12 \mathrm{~h}$ v.o. \\
& Fitak & $1-2$ comp. $/ 24 \mathrm{~h}$ en cursos de 4-5 días consecutivos cada 28 días. 2-4 series v.o. \\
& Fingolimod & Gilenya $^{\circledR}$ & 1 comp. 0,5 mg/24 h v.o. \\
\hline
\end{tabular}

bre de 2009 había 64.600 pacientes tratados con natalizumab, hasta el 9 de febrero de 2010 se han presentado 35 casos confirmados de LMP, lo que supone un riesgo de 0,52 por 1000 pacientes. De los 35 casos han fallecido 8 pacientes. La experiencia indica que el riesgo de LMP aumenta a mayor duración del tratamiento y es bajo en el primer año de infusión.

\section{MITOXANTRONA}

La mitoxantrona ${ }^{10}$ es un fármaco inmunosupresor e inmunomodulador. Es un derivado sintético de la antraciclina que actúa intercalándose en el ADN y rompiendo sus cadenas y las posteriores uniones cruzadas entre ellas.

Ha demostrado reducción en la tasa anual de brotes, una lentificación en la progresión de la enfermedad y una mejoría en los parámetros de RMN. Indicada en EMRR, EMSP y EMPR.

Cuenta con dos potenciales efectos adversos severos: cardiotoxicidad, que puede alcanzar el $5 \%$ cuando se supera una dosis acumulada de $100 \mathrm{mg} / \mathrm{m}^{2}$ y que hace preciso monitorizar la función cardiaca, y leucemia mieloide aguda, independiente de la dosis, con una frecuencia alrededor del $0,25 \%$ y que se puede presentar de forma tardía hasta cinco años después de finalizar el tratamiento.

\section{AZATIOPRINA}

La azatioprina ${ }^{11}$ es un fármaco con efectos inmunosupresores inespecíficos que afecta a la replicación del ADN, por su actividad contra los metabolitos de la purina. Su efecto beneficioso aparece de forma diferida, pasados entres tres y seis meses desde el inicio del tratamiento.

Hasta el año 1995 era el único tratamiento disponible para la EM.

Alternativa en EMRR en pacientes que no toleran o rechazan los fármacos de primera línea, si bien no se dispone de estudios clase I que avalen su eficacia. Es posible que reduzca la tasa de brotes pero no se ha mostrado eficaz para evitar la progresión de la discapacidad.

Tabla 4. Fármacos en distintas fases de ensayos clínicos para el tratamiento de la EM.

\begin{tabular}{|lll|}
\hline Uso previsto & Fármaco & Dosis recomendada \\
\hline Inmunomodulador & Laquinimod & 1 comp. $0,6 \mathrm{mg} / 24 \mathrm{~h}$ v.o. \\
& Teriflunamida & Dosis óptima no establecida. \\
& BG12 & Dosis óptima no establecida. \\
Inmunosupresor & Alemtuzumab & Administración anual i.v. \\
& Rituximab-Ocrelizumab & 2 ciclos de 1 gr/15 días i.v. \\
\hline
\end{tabular}

Sus efectos secundarios más frecuentes son leucopenia reversible, efectos sistémicos y hepatotoxicidad. Puede ser conveniente administrar ácido fólico durante el tratamiento.

\section{OTROS INMUNOSUPRESORES}

Entre los fármacos inmunosupresores ${ }^{12}$ que se utilizan en la actualidad, destacan:

- CICLOFOSFAMIDA: Con propiedades antimitóticas e inmunosupresoras. Ha demostrado un enlentecimiento de la progresión de la enfermedad.

- METOTREXATO: Inmunosupresor que interfiere en la síntesis de ADN con efectos inhibidores sobre la generación de mediadores inflamatorios. Se ha establecido una recomendación de tipo C en EM progresiva.

- CICLOSPORINA: Inmunosupresor que provoca una depleción selectiva de linfocitos T helper. Puede tener algún efecto beneficioso en EM progresiva.

\section{ESTEROIDES}

Se está estudiando su utilización como tratamiento patogénico de la enfermedad, en forma de pulsos periódicos que podrían disminuir la progresión, el volumen lesional y la atrofia cerebral. Ya se está administrando en algunos centros durante el puerperio, por el mayor riesgo de brotes en este periodo.

\section{INMUNOGLOBULINAS}

Se han estudiado en pacientes con CIS, en los que se observó una reducción significativa de la probabilidad de presentar EM clínicamente definida (EMCD).

En EMRR parece que pueden disminuir la tasa de brotes, sin que modifiquen la progresión de la enfermedad.

\section{PLASMAFÉRESIS}

Este tratamiento no está indicado excepto en los brotes refractarios a esteroides.

El inicio precoz de la plasmaféresis ha demostrado mejoría clínica del brote tras la sesión.

Se ha utilizado para la reconstitución inmunológica en casos de LMP en pacientes tratados con natalizumab (aunque puede existir riesgo de aparición de Sd. Inflamatorio de reconstitución inmunológica). 


\section{HACIA UN NUEVO ALGORITMO TERAPEÚTICO}

Actualmente existen múltiples tratamientos en distintas fases de ensayos clínicos, son fármacos de características muy diferentes, con indicaciones aún por determinar, que próximamente modificarán el tratamiento y previsiblemente el curso de la enfermedad.

\section{CLADRIBINA}

La cladribina ${ }^{13}$ o 2 Cloro-2'deoxiadenosina (2-CdA), es un conocido agente antileucémico con administración vía oral, se trata de un análogo clorado de las purinas resistente a la adenosina desaminasa que produce una depleción linfocitaria selectiva, precoz y prolongada (con efecto reducido sobre neutrófilos), con lo que se consigue un efecto muy precoz en disminuir la actividad inflamatoria de la enfermedad.

Es un inmunosupresor que atraviesa la barrera hematoencefálica, inicialmente estaría indicado en EMRR.

Ha demostrado una reducción de la tasa de brotes en un $58 \%$, una disminución de la progresión de la discapacidad de forma mantenida y una caída del $75 \%$ de la actividad inflamatoria de la enfermedad en base a criterios de RMN.

Como efectos adversos cabe destacar: linfopenia (que puede dar lugar a infecciones y tumores) cefalea, nasofaringitis y nauseas.

\section{FINGOLIMOD (FTY720)}

El fingolimod ${ }^{14}$ es un derivado químico del myriocin, un metabolito del hongo Isaria sinclairii, que había sido utilizado en la medicina tradicional china y del que eran conocidos sus efectos agresivos sobre los linfocitos. Fue estudiado inicialmente en la prevención del rechazo en los trasplantes de órganos sólidos. Su administración es por vía oral.

Se trata de un agonista de los receptores de la esfingosina 1-fosfato que, tras unirse a éstos, induce su internalización, de forma que tanto los linfocitos T CD4+ y CD8+ como los linfocitos B quedan «secuestrados» en los órganos linfoides secundarios.

Atraviesa la BHE y accede al SNC con efecto sobre poblaciones neuronales. Bloquea células endoteliales de la barrera hematoencefálica disminuyendo su permeabilidad.

Produce una marcada linfocitopenia periférica pero sin efecto inmunosupresor generalizado.

Disminuye el porcentaje de lesiones captantes de gadolinio en RMN y la tasa anual de brotes en un $60 \%$ respecto al placebo ${ }^{15}$. Actualmente hay ensayos en formas RR (fase III) y PP.

Entre los efectos adversos destacan: bradicardia (en la primera dosis), edema de mácula, nauseas, aumento de las transaminasas e infecciones.

\section{LAQUINIMOD}

El laquinimod ${ }^{16}$ es un fármaco inmunomodulador en desarrollo para EM. Es una quinolona-3-carboxamida con una biodisponibilidad oral excelente. Su mecanismo de acción se basa en la inhibición de citoquinas proinflamatorias y en la activación de citoquinas antiinflamatorias.
Ha demostrado su eficacia con una disminución en un $40 \%$ de las lesiones captantes de gadolinio en RMN. Actualmente están en curso dos estudios en fase III (Allegro y Bravo).

Como efecto secundario más frecuente destaca el aumento de transaminasas.

\section{TERIFLUNAMIDA}

La teriflunamida ${ }^{17}$ es un fármaco inmunomodulador, bloquea la enzima mitocondrial dihidro-oratato deshidrogenasa e inhibe la proliferación de linfocitos $\mathrm{T}$ y B, su metabolito activo es la Laflunamida, usado en el tratamiento de la artritis reumatoide, produce inhibición parcial de la síntesis de primidina y activación de citocinas antiinflamatorias dependientes de IL-1.

Ha demostrado un descenso del $61 \%$ de las lesiones captantes de gadolinio en RMN.

Buen perfil de seguridad, incluso en combinación con IFN. Sus efectos secundarios más frecuentes son: alopecia, nasofaringitis, artralgias e intolerancia digestiva.

\section{BG12}

Se trata de la formulación oral del dimetil-fumarato, un inmunomodulador utilizado en el tratamiento de la psoriasis que actúa de intermediario en el ciclo de Krebs, con efectos antiinflamatorios y citoprotectivos: disminuye el número de linfocitos $\mathrm{T}$, produce una desviación de citocinas de Th1 a Th2, disminuye infiltración de macrófagos, de microglia, de citocinas proinflamatorias y de TNF-1 alfa.

Actualmente existen dos ensayos clínicos en marcha que se encuentran en fase IIB (Confirm, Define).

Ha demostrado una reducción del número total de nuevas lesiones en $\mathrm{RMN}$ (70\% frente a placebo), y una tendencia a la reducción de brotes.

No se han presentado reacciones adversas graves.

\section{ALEMTUZUMAB}

Es un anticuerpo monoclonal humanizado tipo IgG1k que se fija selectivamente sobre la glucoproteína CD52 produciendo una depleción linfocitaria de larga duración. Fármaco aprobado por la FDA para el tratamiento de la leucemia linfocítica crónica de células B.

Tiene como ventaja la administración anual endovenosa.

En formas RR ha demostrado disminución de lesiones captantes de gadolino en RMN y de la tasa de brotes. Suprime la actividad inflamatoria pero no previene la progresión de discapacidad. Actualmente están en curso dos ensayos en fase II.

Sus efectos adversos principales son las reacciones infusionales.

\section{RITUXIMAB-OCRELIZUMAB}

El rituximab ${ }^{18}$ es otro anticuerpo monoclonal, este actúa selectivamente sobre los Linfocitos B que expresan CD20. Existe una amplia experiencia por su uso en otras enfermedades (neoplásicas y 


\section{Nuevas perspectivas en el tratamiento de la esclerosis múltiple}

disinmunes). El ocrelizumab ${ }^{19}$ es rituximab humanizado, más seguro por su menor inmunogenicidad y mejor perfil de efectos secundarios. Existe un estudio clínico en fase II.

$\mathrm{Su}$ eficacia fundamental se basa en el tratamiento de la inflamación. Ha demostrado una disminución precoz del 91\% de las lesiones captantes de gadolinio en RMN y de la tasa de brotes, con un efecto prolongado. En EMPP se han obtenido resultados negativos.

Entre sus efectos adversos cabe destacar: las reacciones infusionales y las de reactivación viral.

\section{NUEVOS TRATAMIENTOS SINTOMÁTICOS}

En un futuro próximo el arsenal terapéutico de la enfermedad va a ser ampliado con la aparición de dos nuevos fármacos, pendientes de su aprobación, destinados al tratamiento sintomático y a conseguir una mejora de la calidad de vida de los pacientes con EM, son Sativex y Fampridina.

\section{SATIVEX ${ }^{\circledR}$}

El principio activo de este nuevo fármaco es el Nabiximol, una combinación de 2,7 mg de tetrahidrocannabinol, con efecto analgésico, relajante muscular, antiemético y estimulante del apetito, junto con 2,5 mg de cannabidiol, que potencia el efecto analgésico, es ansiolítico y anticonvulsivante. Se fija en los receptores cannabinoides CB1 provocando una regulación de neurotransmisores (glutamato y GABA) y CB2 de las células inmunes, produciendo la liberación de citocinas.

Ya cuenta con indicación para dolor neuropático en EM y oncológico en Canadá, solicitada para espasticidad en EM. Se ha utilizado como uso compasivo en 61 países, actualmente hay 68 pacientes con EM tratados en España con este fármaco. Ha demostrado una mejoría significativa del dolor del $41 \%$ y una mejoría de la espasticidad del $40 \%$ con reducción de la frecuencia de los espasmos del $25 \%$.

Se administra en forma de pulverización en la mucosa oral sublingual, de dosificación flexible. Entre sus efectos secundarios destacan: mareo y vértigo, cefalea, fatiga y somnolencia, patología oral (aftas, leucoplasia), trastornos psiquiátricos, taquicardia e hipotensión arterial. Y está contraindicado en esquizofrenia, trastornos psicóticos, trastorno de personalidad, historia de abuso de psicótropos, embarazo, lactancia, menores de 18 años.

\section{FAMPRIDINA}

La 4-aminopiridina ${ }^{20}$, es un alquitrán derivado del carbón mineral, actúa bloqueando los canales de potasio en el SNC aumentando la conducción en los axones desmielinizados produciendo una mejora del impulso nervioso. Se han realizado estudios en otras enfermedades neurológicas como en la miastenia, en la esclerosis lateral amiotrófica (ELA) y en lesiones medulares.

Está solicitada la indicación para trastorno de la marcha en EM, con lo que se convertiría en el primer tratamiento que puede ayudar en la movilidad de los pacientes con EM.
Se estima que la dosis eficaz es de $10 \mathrm{mg}$ dos veces al día vía oral.

Para valorar la eficacia de este nuevo tratamiento sintomático se ha medido el tiempo y la velocidad del paciente para recorrer la distancia de 7,5 metros. Hay aproximadamente un 35\% de respondedores que presentan una mejora de la velocidad de la marcha, la fatiga, la funcionalidad de los miembros inferiores, la espasticidad (medida según la escala de Ashworth) y el balance muscular. Por lo que ha demostrado una mejora de la función motora en EM en los pacientes respondedores, independientemente del tipo de EM y del tratamiento concomitante.

Como efectos secundarios destacan los mareos y la posibilidad de desencadenar crisis epilépticas.

\section{CONCLUSIÓN}

En los próximos años los fármacos disponibles para el tratamiento de la EM van a dar lugar a un nuevo algoritmo terapéutico en la enfermedad, los nuevos fármacos están destinados a mejorar el curso de la enfermedad y la calidad de vida de los pacientes.

Ya contamos con nuevos fármacos que han demostrado su eficacia, tanto modificadores del curso de la enfermedad y como tratamientos sintomáticos, algunos únicamente pendientes de aprobación y que estarán disponibles probablemente a lo largo de 2011, que cuentan con la ventaja añadida de su administración vía oral, pero cuya indicación deberá ser individualizada.

Pero lo más esperanzador es la multitud de estudios clínicos que están desarrollando nuevos fármacos que actúan sobre fases cada vez más específicas de la respuesta inmunitaria, para un mejor conocimiento y control de la enfermedad.

\section{BIBLIOGRAFÍA}

1. Cook, S.D. Handbook of multiple sclerosis. Basel: Marcel Dekker, INC., New York 2001.

2. Sociedad Española de Neurología. Guía oficial para el diagnóstico y tratamiento de la esclerosis múltiple. Prous Science. Barcelona 2007.

3. Gooding, D.S. et al. Disease-modifying therapy in MS: a critical review of the literature. Part I: Analysis of clinical trial errors. Journal of Neurology 2004; 251 Supl 5: 3-11.

4. Barkhof, F. et al. Comparison of MRI criteria at first presentation to predict conversion to clinically definite multiple sclerosis. Brain 1997; 120: 2059-2069.

5. Hernández, M.A. et al. Tratamiento sintomático de la esclerosis múltiple. Revista Española de Esclerosis Múltiple 2007; 4: 5-14.

6. Goodin, D.S. et al. Treatment of multiple sclerosis with human interferon beta. The international MS Journal 2005; 12: 96-108.

7. Johnson, K.P. et al. Copolymer 1 reduces relapse rate and improves disability in relapsing-remitting multiple sclerosis: results of a phase multicenter, doubleblind placebo controlled trial. The Copolymer 1 Multiple Sclerosis Group. Neurology $1995 ; 45:$ 1268-1276.

8. Coyle, P.K. y Jeffery, D.R. Clinical efficacy and benefit of natalizumab. Multiple Sclerosis 2009; 15(S4): S7-S15.

9. Coyle, P.K. et al. Best practice recommendations for the selection and management of patients with multiple sclerosis receiving natalizumab therapy. Multiple Sclerosis 2009; 15(S4): S26-S36.

10. Hartung, H.P. et al. Mitoxantrone in multiple sclerosis: a placebo-controlled, double blind, randomised, multicentre trial. Lancet 2002; 360: 2018-2025.

11. Palace, J., Rothwell, P. New treatments and azathioprine in multiple sclerosis. Lancet 1997; 350:261.

12. Gonsette, R.E. Inmunosuppressants in multiple sclerosis: the past, the present and the future. Revista Española de Esclerosis Múltiple 2008; 6: 11-21. 
13. Giovannoni, G. et al. A placebo-controlled trial of oral cladribine for relapsing multiple sclerosis. New England Journal of Medicine 2010; DOI: 10.1056/NEJMoa0902533

14. Kappos, L. et al. Oral fingolimod (FTY720) for relapsing multiple sclerosis. New England Journal of Medicine 2006; 355: 1124-1140.

15. Cohen, J.A. et al. Oral fingolimod or intramuscular interferon for relapsing multiple sclerosis. New England Journal of Medicine 2010; 362: 402-415.

16. Polman, C. et al. Treatment with laquinimod reduces development of active MRI lesions in relapsing sclerosis multiple. Neurology 2005; 64: 987-991.

17. O'Connor, P.W. et al. Teriflunomide Multiple Sclerosis Trial Group; University of British Columbia MS/MRI Research Group. A phase II study of the safety and efficacy of teriflunomide in multiple sclerosis with relapses. Neurology 2006 66:894-900.

18. Hauser, S.L. et al. A phase II randomized, placebo-controlled, multicenter trial of rituximab in adults with relapsing remitting multiple sclerosis. Neurology 2007 68 Supl 1: A99.

19. Hauser, S. et al. B-cell depletion with an anti-CD20 monoclonal antibody (rituximab) in relapsing remitting multiple sclerosis. New England Journal of Medicine 2008; 358: 676-688.
20. Goodman, A.D. et al. Sustained-release oral fampridine in multiple sclerosis: a randomized, double-blind, controlled trial. Lancet 2009; 373: 732-738.

21. Hirst, C.L. et al. 1-H treatment in patients with aggressive relapsing remitting multiple sclerosis. Journal of Neurology 2008; 255: 231-238.

22. Rieckman, P. et al. Correlation of soluble adhesion molecules in blood and cerebrospinal fluid with magnetic resonance imaging activity in patients with multiple sclerosis. Ann Neurology 1998; 43: 384-387.

23. Jimenez, M.D. Esclerosis Múltiple. Continua Neurológica. Sociedad Española de Neurología. Ars Medica, Barcelona 2007.

24. O'Connor, R.P. et al. Efficacy of intramuscular interferon beta-1a in patients with clinically isolated syndrome: analysis of subgroups based on new risk criteria. Multiple Sclerosis 2009; 15: 728-734.

25. Vollmer, T. et al. Acetato de glatiramero tras el tratamiento de inducción con mitoxantrona en la esclerosis múltiple recidivante. Multiple Sclerosis 2008; 14: 663670

26. Arroyo, R. y De las Heras, V. Esclerosis múltiple: actualización del tratamiento. Mayo ediciones. Barcelona 2009.

27. Ruiz-Peña, J.L. y Izquierdo-Ayuso, G. Inmunosupresores y esclerosis múltiple. Revista Española de Neurología 2002; 35: 373-380. 